\title{
SATELLITE-BASED RADAR MEASUREMENTS FOR VALIDATION OF HIGH- RESOLUTION SEA STATE FORECAST MODELS IN THE GERMAN BIGHT
}

\author{
A. Pleskachevsky a,*, C. Gebhardt ${ }^{\text {a }}$, W. Rosenthal ${ }^{\text {a }}$, S. Lehner ${ }^{\text {a }}$, \\ P. Hoffmann ${ }^{\text {b }}$, J. Kieser ${ }^{b}$, T. Bruns $^{b}$, A. Lindenthal ${ }^{\text {c }}$, F. Jansen ${ }^{c}$, A. Behrens ${ }^{d}$ \\ ${ }^{a}$ German Aerospace Center (DLR), Remote Sensing Technology Institute, Maritime Security Lab, \\ Henrich-Focke-Straße 4, 28199 Bremen, Germany - Andrey.Pleskachevsky@dlr.de, Claus.Gebhardt@dlr.de, \\ Wolfgang.Rosenthal@dlr.de, Susanne.Lehner@dlr.de \\ ${ }^{\mathrm{b}}$ German Meteorological Service (DWD), \\ Bernhard-Nocht-Str. 76, 20359 Hamburg, Germany - Peter.Hoffmann@ dwd.de, Jens.Kieser@dwd.de, Thomas.Bruns@dwd.de \\ ${ }^{\mathrm{c}}$ German Maritime and Hydrographic Agency (BSH), \\ Bernhard-Nocht-Str. 78, 20359 Hamburg, Germany - Anja.Lindenthal@bsh.de, Frank.Janssen@bsh.de \\ ${ }^{\mathrm{d}}$ Helmholtz Center Geesthacht (HZG), Institute of Coastal Research, \\ Otto-Hahn str.1, 21502 Geesthacht, Germany - Arno.Behrens@hzg.de
}

KEY WORDS: Ocean waves, coastal processes, wave modeling, high resolution, remote sensing, radar satellites

\begin{abstract}
:
Remote sensing Synthetic Aperture Radar (SAR) data from TerraSAR-X and Tandem-X (TS-X and TD-X) satellites have been used for validation and verification of newly developed coastal forecast models in the German Bight of the North Sea. The empirical XWAVE algorithm for estimation of significant wave height has been adopted for coastal application and implemented for NRT services. All available TS-X images in the German Bight collocated with buoy measurements (6 buoys) since 2013 were processed and analysed (total of 46 scenes/passages with 184 StripMap images). Sea state estimated from series of TS-X images cover strips with length of $\sim 200 \mathrm{~km}$ and width of $30 \mathrm{~km}$ over the German Bight from East-Frisian Islands to the Danish coast. The comparisons with results of wave prediction model show a number of local variations due to variety in bathymetry and wind fronts.
\end{abstract}

\section{INTRODUCTION}

\subsection{Novel satellite borne SAR for oceanography}

The estimation of marine and meteorological parameters is an important task for operational oceanographic services. In comparison to in-situ buoy measurements at a single location, remote sensing allows to cover large areas and to estimate the spatial distribution of investigated characteristics. The spatial validation of forecast data, e.g. sea state and surface wind, through remote sensing data can visibly improve the forecast quality and help explain natural phenomena beyond ordinary circumstances like storm front propagation, gusts and emergence of wave group with extreme wave height (Lehner et al., 2012, 2013, Pleskachevsky et al., 2012).

Space borne SAR (Synthetic Aperture Radar) as a remote sensing instrument is a unique sensor which can cover large areas and provide two dimensional information of the ocean surface. Due to its high resolution, daylight and weather independence and global coverage, space borne SAR sensors of the latest generation are particularly suitable for ocean and coastal applications. Over the last years, a number of new high resolution X-band radar satellites have been launched, which provides the possibility to image and measure the sea surface with high resolution e.g. TerraSAR-X (TS-X), TanDEM-X(TD$\mathrm{X})$ and COSMO-Skymed satellites. This opens a new perspective for investigating sea state and connected processes in coastal areas, where spatial variability in sea surface play an important role. A wide spectrum of features and signatures of sea state and derived parameters are simultaneously involved and can be observed in high resolution images including surface wind and gusts, individual waves and their refraction, wave braking effects, etc. Knowledge of basic geophysical processes and its imaging mechanism is necessary for successful processing of images and for organization of NRT services to provide the information to interested users like the German Meteorological Service (Deutscher Wetterdienst, DWD).

\subsection{Objectives: improving of sea state prediction in German Bight and intertidal zones of North Sea}

The German Bight in the North Sea is characterized by a strong dependence on tides in complex topography with number of islands and shoals. Fast-changing of water levels and local currents reaches more than $2 \mathrm{~m} \cdot \mathrm{s}^{-1}$ as well as emergence of numerous sandbars and shoals greatly influences the wave propagation.

The scientific and technical tasks presented in this paper were on the one hand the improvement of forecast services especially in coastal areas by development of high resolution coastal model (1). On the other hand, new algorithms were to be developed for estimation of meteo-marine parameters from SAR images in coastal waters with high accuracy (2) and likewise products were to be conceived for NRT services (3). As a result an automatic chain "Coastal waves prediction/NRT SAR acquisition and processing/delivery on DWD and validation" was developed.

In order to improve forecast accuracy in coastal areas and more accurate mapping of coastlines (1) and coupled to a circulation model (2), the prediction model from DWD was equipped with a new grid with spatial resolution of $0.5 \mathrm{~nm}(\sim 900 \mathrm{~m})$. The wave model was coupled to the circulation model of the BSH (German Maritime and Hydrographic Agency) uses the same bathymetry grid. The purpose of this modification was to improve the forecast quality especially near the coast, where the bathymetry varies greatly spatially and currents and water level vary temporally. The users (e.g. shipping company) had requested an improvement of sea state prediction in the significant wave height $H_{S}$ domain $0.5 \mathrm{~m}-1.5 \mathrm{~m}$. This is due to 
the fact that disembarking height from a boat onto an offshore construction (e.g. wind farm) may not exceed $1.2 \mathrm{~m}$. In case $H_{S}$ exceeds $1.2 \mathrm{~m}$, the disembarking is too hazardous for crew members and the transport ship must return back to the harbour. Such works are planned in advance and inaccurate predictions cause high additional costs.

\subsection{Coastal processes and forecast services}

The wave models of the third generation have been developed and used for sea state prediction, e.g. WAVEWATCH III used by NOAA (http://polar.ncep.noaa.gov/waves/) and UKMET (United Kingdom Meteorological Office), and WAM model used by forecast services in Europe like ECMWF (European Center of Medium range Weather Forecast), the German Weather Service (DWD, http://www.dwd.de), and the Danish Meteorological Institute (DMI, www.dmi.dk), which are a part of the global marine weather and warning systems. The forecasts are accessible also for public users by MF (Meteo France, http://marine.meteofrance.com/marine/).

Uncertainties are to be taken into account when considering coastal areas: wind input implies well-timed atmospheric front propagation and interaction in shallow water caused between waves, currents and the sea bottom becomes important. Currents and the sea bottom cause waves to refract, get dampened, become steeper and break. The wave energy dissipates and transfers into turbulence and acceleration of flow currents (radiation stress).

The high resolution Coastal Wave Model (CWAM) for the German Bight and the western Baltic Sea has been developed by DWD and BSH in cooperation with the Helmholtz-Zentrum Geesthacht (HZG). CWAM is based on the Wave Model (WAM) and will complement the series of wave models consisting of the Global Wave Model (GWAM) and the European Wave Model (EWAM) which are operated by the DWD. CWAM runs in a pre-operational state for an extended forecast time range of 48 or 72 hours (Kieser et al., 2013).

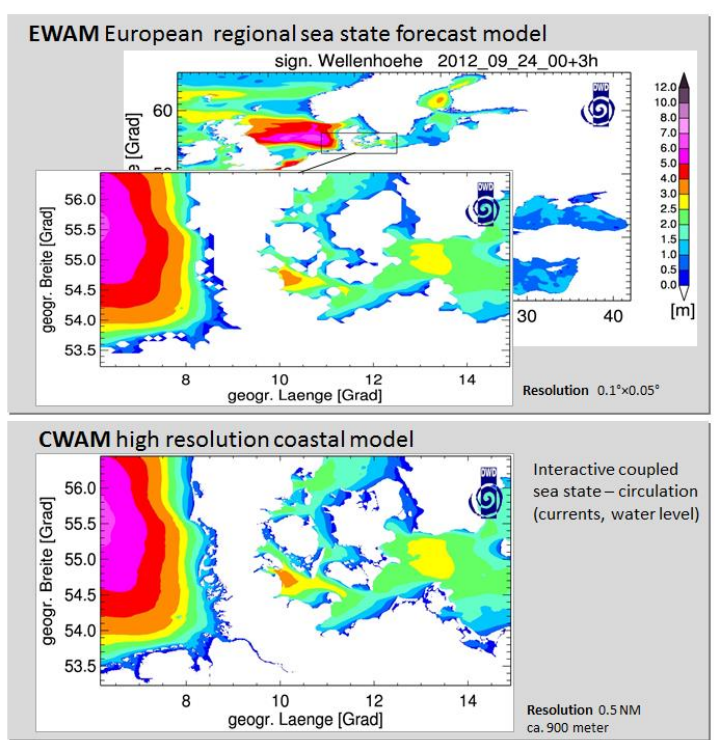

Figure 1. Conventional EWAM sea state forecast and new high resolution coastal model CWAM $(0.5 \mathrm{NM}=\sim 900 \mathrm{~m})$. Local differences of resulting $H_{S}$ in coastal areas are in the range of meters because of more structures in bathymetry (1) and rapid change of water level and circulation currents by tides not resolved by EWAM in intertidal Watt zone (2).
A product combines forecasts and a near real time assessment with high-resolution satellite data. Local differences of resulting $H_{S}$ EWAM/CWAM in coastal areas are in the range of meters because of more structure in bathymetry (1) and rapid change of water level and circulation currents by tides (2) (see Fig.1).

\section{REMOTE SENSING NEW TECNIQUE}

\subsection{Satellite borne SAR data for oceanographic applications}

In this paper the data from TerraSAR-X (TS-X) and its twin TanDEM-X (TD-X) were used for validation. The X-band SAR satellite TS-X (launched in 2007) and its twin TD-X (launched in 2009) operates from $514 \mathrm{~km}$ height at sun-synchronous orbit with ground speed of $7 \mathrm{~km} \cdot \mathrm{s}^{-1}$ (15orbits per day). It operates with a wavelength of $31 \mathrm{~mm}$ and a frequency of $9.6 \mathrm{GHz}$. The repeat-cycle is 11 days, but the same region can be imaged by different incidence angles after three days dependent on scene latitude. Typical TS-X incidence angles range between $20^{\circ}$ and $55^{\circ}$.

TS-X operates five different basic imaging modes with different spatial resolution: from High-Resolution Spotlight $5 \mathrm{~km} \times 10 \mathrm{~km}$ to about $280 \mathrm{~km} \times 400 \mathrm{~km}$ wide ScanSAR mode with $40 \mathrm{~m}$ resolution. For sea state analysis, the StripMap mode with $30 \mathrm{~km} \times 50 \mathrm{~km}$ and $3 \mathrm{~m}$ resolution is most suitable. On the one hand, the waves are well imaged, and other hand, the image footprint allows spatial analysis of sea sate. E.g. ScanSAR mode, which covers more than $100 \mathrm{~km}$ strip with $18 \mathrm{~m}$ resolution, allows analysis of long swell waves only and is therefore not suitable for coastal applications.

Methods to derive wind speed and sea state using simple empirical models from high-resolution SAR data have been developed by DLR: a new nonlinear wind algorithm XMOD-2 (Li and Lehner, 2013) and a new empirical model function XWAVE-2 (Lehner et al., 2013) for obtaining significant wave height from X-band data. Both algorithms are capable of taking into account fine-scale effects in coastal areas. As the sea state is directly connected to local wind, the wind information form the same SAR image will be taken for filtering of noise and checking of sea state. For coastal areas, the sea state algorithm was adapted and tuned again to account for non-standard coastal effects.

\subsection{Surface wind from TerraSAR-X data}

The SAR wind field retrieval approach was first developed for C-band SAR provided by, for example, ERS-2 and ENVISAT ASAR. These approaches utilized an empirically derived Geophysical Model Functions (GMF) that related local wind conditions and sensor geometry to radar cross section values (e.g. CMOD4 or CMOD5). To utilize the new SAR systems, an $\mathrm{X}$-Band linear algorithm XMOD-1 and later a nonlinear XMOD-2 was established for $\mathrm{VV}$ and $\mathrm{HH}$ polarized data to obtain the wind fields ( $\mathrm{Li}$ and Lehner, 2013). The relationship between X-band radar cross section and wind speed, wind direction and incidence angle in XMOD-2 is given by:

$$
\sigma_{o}(U, \theta, \varphi)=B_{0}^{p}\left(U_{10}, \theta\right)\left(1+B_{1}\left(U_{10}, \theta\right) \cos (\phi)+B_{2}\left(U_{10}, \theta\right) \cos (2 \phi)\right)
$$

where $\sigma_{o}$ is NRCS, $U_{10}$ is the wind speed, $\phi$ is the wind relative direction. Eq. 1 is applicable for an incidence angle $\theta$ between $20^{\circ}$ and $60^{\circ}$ and for wind speed from $2 \mathrm{~m} \cdot \mathrm{s}^{-1}$ to $25 \mathrm{~m} \cdot \mathrm{s}^{-1}$. The parameters $B_{i}$ with $i=0,1,2$ are tuned using the measurement data sets (Li and Lehner, 2013). To determine wind direction, streak structures on the sea surface of the image are used. These are 
produced by airflow turbulent eddies at the boundary. Shadows behind the coast also give evidence of wind blowing from the coast.

Data from the Spaceborne Imaging Radar-C/X (SIR-C/X) mission in 1994 and from the European Center (ECMWF) reanalysed wind fields ERA-40 (ECMWF Re-Analysis of the global atmosphere and surface conditions for 45-years) were used to tune the algorithm. The results were validated using insitu measurements from collocated buoys and modelled data with different resolution (HIRLAM model and DWD COSMO).

The wind field can be retrieved with $20 \mathrm{~m}$ resolution by the XMOD algorithm for TS-X images. In contrast to the previously developed XMOD-1, XMOD-2 consists of a set of nonlinear GMFs and therefore depicts the difference between upwind and downwind of the sea surface backscatter in X-band SAR imagery. By exploiting 371 collocations with in situ buoy measurements which are used as the tuning dataset together with analysis wind model results, the retrieved TS-X/TD-X sea surface wind speed using XMOD-2 shows a close agreement with buoy measurements with a bias of $-0.32 \mathrm{~m} \cdot \mathrm{s}^{-1}$, an RMSE of $1.44 \mathrm{~m} \cdot \mathrm{s}^{-1}$ and a scatter index (SI) of $16.0 \%$. Further validation using an independent dataset of 52 cases shows a bias of $0.17 \mathrm{~m} \cdot \mathrm{s}^{-1}$, an RMSE of $1.48 \mathrm{~m} \cdot \mathrm{s}^{-1}$, and SI of $17.0 \%$ comparing with buoy measurements (Li and Lehner, 2013).

\subsection{Sea state from TerraSAR-X data}

Several retrieval algorithms to derive the two-dimensional ocean wave spectrum or integral wave parameters from SAR data have been developed. As the ocean surface gravity waves are moving targets, the mechanism of their SAR backscatter consist of the linear transformation of tilt and hydrodynamic modulation, as well as the non-linear distortion. This leads, among other effects, to image smearing and to a loss of information beyond the so-called azimuth cut-off wavelength. For TerraSAR-X images this cut-off is about $30 \mathrm{~m}$ for range traveling waves, and the non-linear effects like velocity bunching are visibly reduced in comparison with earlier SAR mission due to lower orbit of $500 \mathrm{~km}$ (e.g. ASAR with $800 \mathrm{~km}$ orbit).

An empirical XWAVE-1 model for obtaining integrated wave parameters has been developed for X-band. The algorithm is based on analysis of image spectra and uses parameters fitted with collocated buoy data and information on spectral peak direction and incidence angle. The new developed XWAVE-2 algorithm to derive significant wave height directly from TS-X SAR image spectra (no transferring into wave spectra) is presented by the equation:

$$
H s=c_{1} \sqrt{E_{I S} \cdot \tan (\theta)}+c_{2} U_{10}+c_{3}+c_{4} \cos (\alpha)
$$

where $\alpha$ is the wave peak direction related to the azimuth direction $\left(0^{\circ} \leq \alpha \leq 90^{\circ}\right)$. The cosine function in the formula describes the dependence of wave peak direction in the image relative to satellite direction, $E_{I S}$ is the integrated value of the directional wave number Image Spectrum (IS). Parameters $c_{1}-c_{4}$ are the coefficients tuned to various data sets and dependent on incidence angle $\theta$. They are determined from a linear fitting between $E_{I S}$ and the collocated significant wave height, computed by the DWD wave model, collocated buoy measurements, WaMoS-II (Wave Monitoring System) and Radar altimeter data (Bruck and Lehner 2013). The integration domain chosen is limited by minimal and maximal wavelength in order to avoid the effects of wind streaks by turbulent boundary layer and the "cut-off" effect of SAR imaging of short sea surface waves. The values are set to $L_{\min }=30 \mathrm{~m}$ and $L_{\max }=600 \mathrm{~m}$, which corresponds to $k_{\max }=0.2$ and $k_{\min }=0.01$ in deep water. The TS-X Image Spectrum IS(k) is obtained through the Fast Fourier analysis (FFT) done on a subscene of the radiometrically calibrated TS-X/TD-X intensity image. Generally, the algorithm has been tuned with the NOAA buoys in open ocean where hundreds of TS-X scenes were acquired especially for this purpose with scatter index SI for significant wave height $\mathrm{SI}_{\mathrm{Hs}}{ }^{\mathrm{XWAVE} / \mathrm{BUOY}}=0.21$ and $\mathrm{SI}_{\mathrm{L}}{ }^{\mathrm{XWAVE} / \mathrm{BUOY}}=0.13$ for wavelength (Lehner et al., 2013).

\subsection{Improving of sea state algorithm for coastal areas}

The XWAVE algorithm developed for open sea works well for several sub-scenes with well-pronounced waves checked by a homogeneity test. For space-coverage analysis of scenes and automatic NRT services it was necessary to develop and add the series of filters and corrections. A direct implementing of GMF Eq. 2 in coastal area leads to more than $50 \%$ errors of processed data with outliers in the range of meter for $H_{S}$. The sources of error are in first place artefacts in SAR imagery typical for Wadden/mudflat coastal waters: sand banks, wave breaking, ships, current boundaries and atmospheric fronts and even internal waves structures. All these objects impact the image spectra. Such spectral perturbations result in an integrated value which yields a contribution to total energy that is not connected to sea state (Fig.2).

The core of the newly developed Sea Sate Processor (SSP) for both HH and VV polarisation consists of (see Fig.3):

- Step-1: sub-scene pre-filtering (remove image intensity outliers like ships, buoys etc. based on local intensity statistics)

- Step-2: calculation of XMOD-2 wind,

- Step-3: spectral analysis of subscene (FFT, integration and spectral parameters e.g. noise level),

- Step-4: new XWAVE-2 GMF (SSP core),

- Step-5: checking of results using wind speed and integrated spectral parameters (e.g. long structures like sand banks produce high spectral values in domain $k<0.01$ and can be separated).
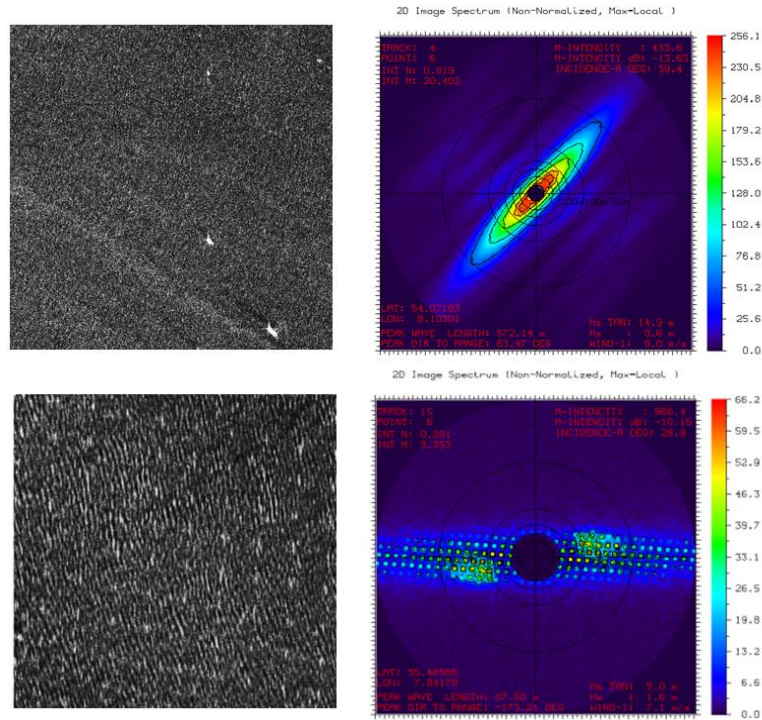

Figure 2. Examples of artefacts in TerraSAR-X sub-images (left) influence spectra (right) and consequently integrated energy: ships (above) and wind park (below). Without prefiltering of such artefacts, wave height can be estimated several times higher than the surrounding $H_{S}$ values. 


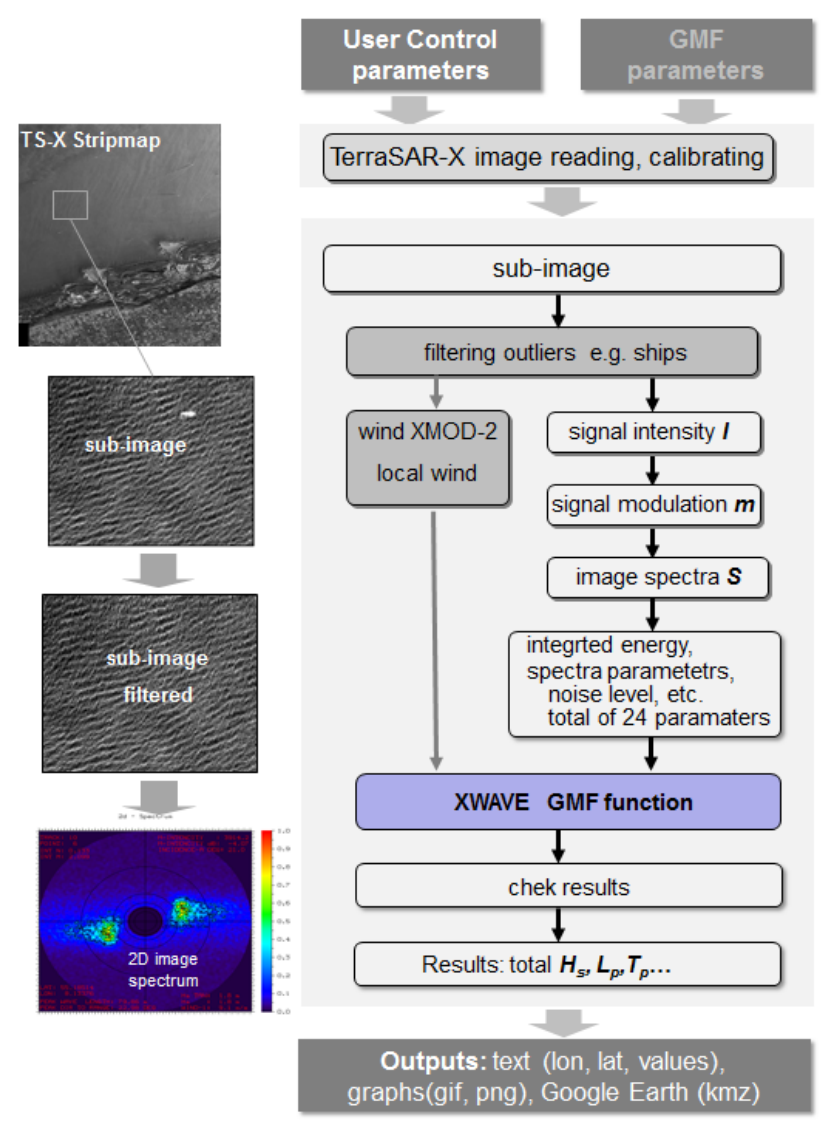

Figure 3. Flow chart of Sea Sate Processor (SSP).

The XWAVE algorithm Eq.2 has been repeatedly validated and adapted for coastal sea state in order to improve the existing function. In the first instance the CWAM model data were used for spatial-temporal validation in order to understand the source of outliers and create the additional terms necessary to compensate perturbations. Model data served thus only to establish an appropriate ansatz function, that is capable of representing all spectrally significant phenomena. Although the model reproduces the spatial distribution of sea sate correctly, the model scatter index for buoy positions and TS-X images date is $\mathrm{SI}_{\mathrm{Hs}} \mathrm{CWAM}^{\mathrm{B} O U Y}=0.23$ and the values are sometimes overestimated by $0.5-1.0 \mathrm{~m}$ (total mean value of $H_{S}=1.5 \mathrm{~m}$ for buoy locations with mean value of $2.5 \mathrm{~m}$ for storm conditions). In a second step, re-tuning of coefficients of the ansatz function (found in the previous step) in order to fit this function to the buoy measurements have been done.

To compare TS-X data with model results, TS-X scenes were processed with $3 \mathrm{~km} \times 3 \mathrm{~km}$ step $(\sim 10$ sub-scenes for range and $\sim 15$ sub-scenes for azimuth directions $10 \times 15=150$ sub-scenes per StripMap image with squares of $\sim 5 \mathrm{~km} \times 5 \mathrm{~km})$. In total, 21 data sets were used (TS-X and CWAM model results at the same day, time differences in order of maximal 10min between model output and TS-X acquisition time). The comparison was made for NN/MSL (normal null/mean sea level) depths $>5 \mathrm{~m}$ in order to exclude dry sand banks during ebb and has about 9000 collocated data pair TS-X/CWAM.

After comparison, XWAVE_C (Coastal) function Eq.2 is: $H s^{X W A V E_{-} C}=a_{1} \sqrt{B_{1} E_{I S} \tan (\theta)}+a_{2} B_{2}+a_{3} B_{3}+a_{4} B_{4}+a_{5} B_{5}$

where $a_{1}-a_{5}$ are coefficients (constants) and $B_{1}-B_{5}$ are functions of spectral parameters. $B_{I}$ represents noise scaling of total energy $E_{I S}$ (short wind waves and their breaking produce an

additional noise that influences non-linearly resulting energy), $a_{2} B_{2}$ term represents wind impact according to the original XWAVE Eq.2. The terms $a_{3} B_{3}$ and $a_{4} B_{4}$ are corrections for eliminating the impact of short (e.g. wave breaking induced) and long (e.g. wind streaks) structures in the SAR image. These structure results in spectral peaks with modes not directly connected to sea state. $a_{5} B_{5}$ is a correction for outliers produced by extra-large structures like sand banks or ship wakes which have not been filtered by Step- 1 .

During data tuning it became clear that not every TS-X scene shows well-pronounced sea state like swell waves and more than 50\% of images cannot be used in the traditional way using Eq.2. However, now using the improved function Eq.3 already a small signal in the SAR-image (no wave-like shaped structures but only speckle), e.g. during calm weather condition $\sim 2 \mathrm{~m} \cdot \mathrm{s}^{-1}<U_{10}<8 \mathrm{~m} \cdot \mathrm{s}^{-1}$ is enough to deduce the sea state that is responsible for noise occurrence in SAR image. More precisely, a large number of wave crests, which are small moving targets, may be not imaged individually by SAR in original shape, but jointly produce radar echo with intensity proportional to their speed and amplitude. Fig.4 demonstrates two examples of different sea state at the same position near buoy "Elbe". The subscenes were shown near the buoy location. The values of spectral maxima differ by orders of magnitude. The storm acquisition on 09.12.2011 at 05:52 shows well pronounced swell waves. Under lower wind condition on 07.01.2015 at 17:19, wave-like structures are hardly visible. Nerveless $H_{S}$ can be accurately estimated based on noise properties of spectra and wind information.

\subsection{Sea state NRT Services}

The Sea Sate Processor (SSP) which includes the XWAVE algorithms was extended and adapted for automated NRT services with 40 input control parameters (FFT size, raster analysis step, ship filtering properties, etc.). The SSP was installed at the Ground Station "Neustrelitz" to provide an operational service and is currently being tested. The delivery of

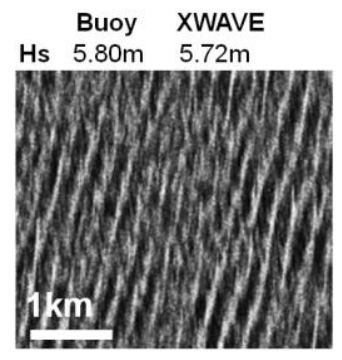

Buoy XWAVE
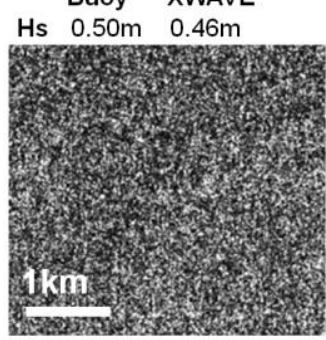

Figure 4. Two examples of different sea state at the same position buoy "Elbe". TerraSAR-X storm acquisition on 09.12.2011 at 05:52: long waves are well pronounced. Low wind condition on 07.01.2015 at 17:19: wave-like strictures are hardly visible, nevertheless, $H_{S}$ can be accurately estimated based on noise properties of spectra and wind information. 
NRT products from "Neustrelitz" to the user (e.g. DWD) occurs by E-mail. The attachment includes a text file (.txt) with the data (lon, lat, $H s)$, the Google Earth file $(. \mathrm{kmz})$ for an illustration of an image file (.jpg).

The program interface allows the user to change the preinstalled properties of automatic data processing and output. The output writing of four text-file types is possible with additional graphic view as TIFF files for processed image, subscenes, 2-D and 1-D spectra (optional):

- TSX_acquisitiondate_mean-values.txt contains the mean values of all parameters for the scene ( $H s$, wind, intensity, incidence angles, geo-coordinates, etc.);

- TSX_acquisitiondate_waves.txt contains only $H s$ with details of the geo-coordinates (for extern users);

- TSX_acquisitiondate_results.txt - all parameters such as integrated energy, noise and other details for each analysed subscene are stored.

- TSX_acquisitiondate_special-points.txt contains information for minimal distance of subscenes analysed to special points (e.g. buoys and offshore construction) specified in input file spetial-points_input.txt (for the German Bight, 6 spatial points were used: measurement piles, wave rider buoys, see Fig.6).

\section{RESULTS}

\subsection{Data ordering, acquisition and processing}

The CWAM model runs at the DWD since 01.01.2013. For validation of XWAVE, a series of images was ordered over the German bight in such a way as to cover the German Bight coastal areas and measurement buoys (see Fig.5): The scenes were ordered twice in series of 3 months for each possible overflight (or each 2/3 day). Additionally, individual images were ordered in order to cover interesting weather conditions in coastal areas and over offshore wind parks (e.g. over FiNO-1 research platform). A series of images ordered by other users were downloaded from the EOWEB (Earth Observation Web) catalogue (e.g. for ship detection, oil detection or wind analysis for wind parks).

When ordered, only $30 \%$ of the ordered scenes were actually acquired. This is due to the low priority of the science-user. In 2015 , this ratio should improve due to installation of a new receiving antenna at the ground station "Neustrelitz".

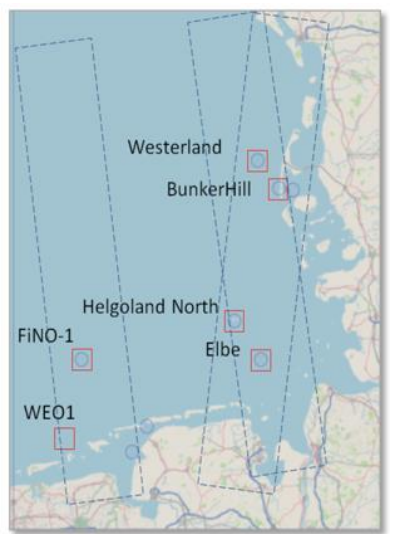

Figure 5. Concept for TerraSAR-X scenes ordering for CWAM wave model validations: two typical ascending and descending overflights collocated with 6 available buoys in the German Bight in wave model domain. Each acquired scene consists of 3-6 single images.
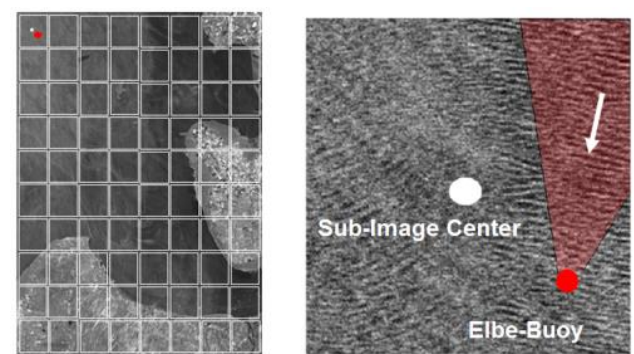

Figure 6. Uncertainties for comparison of TS-X derived sea state and buoy: TerraSAR-X Stripmap typical analysis with subscenes (left) and subscene with buoy (right); buoy represents statistics of a sector with sea state propagating to buoy (red marked area) integrated over time (typically 20min time series). TS-X represents "frozen" $H_{S}$ from spatial (snap shot) statistics of sub-image which includes more spatial variability than buoy data.

As a result, in 2013-2014 a total of 43 TS-X buoy-collocated scenes in the German Bight with 154 images are available and used. In March 2015, this number increased to 46 Scenes with 173 images. However, the data since October 2014 were not anymore included for XWAVE tuning but are only used to verify the statistics.

TS-X scenes were processed with step of $3 \mathrm{~km} \times 3 \mathrm{~km}$ $(\sim 10 \times 15=\sim 150$ subscenes per image). The collocations were done with a time window of $+/$ - 10min for comparison with model data and $+/-20 \mathrm{~min}$ for buoys (slightly varying recording period). CWAM hourly output is at 6:00 and 18:00 UTC and acquisition time for TS-X is 05:50-06:10 for descending orbit and 17:50-18:10 for ascending orbit in German Bight. Spatial collocation occurred in a domain up to $5 \mathrm{~km}$ (subscene size).

A source of uncertainties for comparison lies in the fact that a buoy represents statistics of a small sector of sea state propagating towards the buoy, integrated over time (typically 20min time series). TS-X represents $H_{S}$ from statistics of a wider "frozen" area (snap shot) which includes more variability than buoy data due to e.g. bathymetry disturbing the homogeneity of sea state. As a result, $H_{S}^{\text {buoy }}$ and $H_{S}{ }^{T S X}$ are the same only in case no temporal $(\sim 20 \mathrm{~min})$ and no spatial variations $(\sim 5 \mathrm{~km} \times 5 \mathrm{~km})$ occur in sea state statistics (see Fig.6).

\subsection{Statistics}

Local comparison TS-X/Buoy was conducted for 6 stations in the German Bight (Fig.5). Actually, a total of 46 TS-X Scenes (events) with 173 Images and 72 buoy collocations (collocation around $20 \mathrm{~min}$ and up to $5 \mathrm{~km}$ spatially) were available and analyzed. The scatter index $\mathrm{SI}^{\mathrm{TSX} / \mathrm{BUOY}}=20 \%$ was obtained for all data. Currently the scatter index (the same buoys) for DWD coastal wave model has the same value $\mathrm{SI}^{\mathrm{CWAM} / \mathrm{BUOY}}=20 \%$. However, the model tends to overestimate $H_{S}$ while TS-X values are scattered more symmetrically around the buoy values. Fig.7 and Fig.11 show the same comparison of TS-X XWAVE_C/Buoy measurements in the form of a scatter-plot for all data and a bar-plot for 2013-2014.

Statistical analysis shows the averaged value of collocated $H_{S}$ to be about $1.5 \mathrm{~m}$ in the German Bight. Acquisition with $H_{S}>2.5 \mathrm{~m}$ occurred quite rarely. It was only two times possible to acquire a storm with $H_{S}>5 \mathrm{~m}(09.12 .2011$ and 10.12 .2014$)$. For this reason also the storm on 09.12.2011 in the North Sea was included in the data set, although no CWAM coastal model runs were available for this time. 


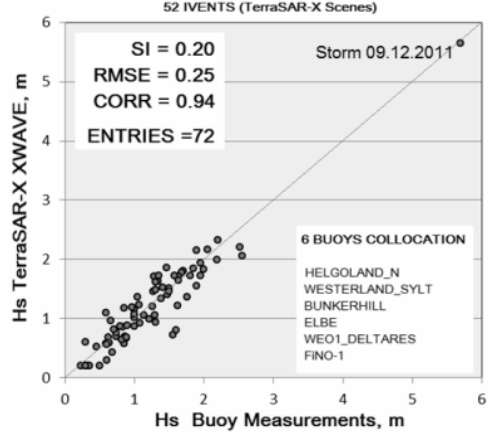

Figure 7. Total comparison of all available data in German Bight 2013-2015 including a storm on 09.12.2011: 46 TerraSAR-X Scenes (overflights/events/days) with 173 Images and 72 buoy collocations (collocation around $20 \mathrm{~min}$ and up to $5 \mathrm{~km}$ spatially).

It is interesting to note that according to earlier studies at HZG in the German Bight generally only $0.3 \%$ of all cases the $H s$ value exceeds $2.5 \mathrm{~m}$ for local wind $U_{10}<12 \mathrm{~m} \cdot \mathrm{s}^{-1}$. This statistics is based on 10-yers sea state reanalysis with the WAM model for the North Sea for 1990-2000 and show a strong dependence of sea state on local wind.

As mentioned above, the spatial comparison TS-X/CWAM model results was done for 21 collocated CWAM data sets (days) with 64 TS-X images and 83664 collocated $H_{S}$ pairs $H_{S}{ }^{\mathrm{TSX}} / H_{S}{ }^{\mathrm{CWAM}}$ (depths $>5 \mathrm{~m}$ ) (Tab.1). The scatterplot is shown in Fig.8. The scatter index amounts to $\mathrm{SI}^{\mathrm{TSX} / \mathrm{CWAM}}=0.28$ and is higher than $\mathrm{SI}^{\mathrm{TSX} / \mathrm{BUOY}}=0.20$ for comparisons TS-X/Buoys. This value was previously 0.23 by tuning with CWAM results only. However, for this case, the TS-X/Buoy comparison was not satisfactory (inaccuracy of the model includes $\mathrm{SI}^{\mathrm{CWAM} / \mathrm{BUOY}}$ $=0.23$ for buoy locations). Thus the coefficients of XWAVE_C GMF were later adapted in order to shift the ansatz function without changing the generic ansatz function type developed before by using CWAM model data only. In Fig.8, it is clearly visible and individual scenes can be separated as "clusters". An analysis of surrounding parameters reveals that the overestimation of forecasted $H_{S}{ }^{C W A M}$ is often connected to overestimated local wind. The atmospheric fronts and gusts (wind input from Atmospheric Model) enter evidently the area with a time delay. The second source of overestimation of sea state in the costal model can be explained by local bathymetry influence. The soft seabed topography in German Bight can change fast due to storms so that model topography can be out of date.

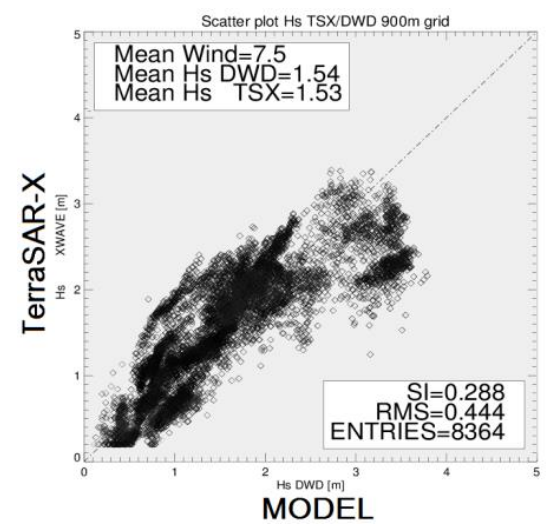

Figure 8. Total comparison of data set Tab.1 TerraSAR-X /CWAM in German Bight 2013-2014: 21 TerraSAR-X Scenes (events) with 64 images and 8364 collocation pairs (TS-X processing with a raster step of $3 \mathrm{kmx} 3 \mathrm{~km}$ and for depth $>5 \mathrm{~m}$ MSL).

\subsection{Examples}

Spatial comparison displays generally correlated sea state spatial distribution (pattern) by TS-X derived data and CWAM model data. The differences are often visible as a local variation of sea state generated by time-shifted wind front (model input hourly) and/or over misplaced sand banks (model bathymetry resolution 900m). Fig.12 presents a spatial validation of a descending TS-X scene consisting of 5 images on 25.12.2013 at 05:59 UTC. The middle sub-figure shows TS-X XWAVE_C values plotted over CWAM model results at 06:00 UTC. The model shows a front with $H_{S}>2 \mathrm{~m}$ (red color) at the Danish coast, corresponding with wind $\sim 11 \mathrm{~m} \cdot \mathrm{s}^{-1}$. TS-X XWAVE_C $H_{S}$ values are about $1.5 \mathrm{~m}$ with local wind $\sim 8 \mathrm{~m} \cdot \mathrm{s}^{-1}$. With a moving wind front and averaged seas state propagation speed of $10 \mathrm{~m} \cdot \mathrm{s}$ ${ }^{1}$, it can cross the area covered by the TS-X image (30km streak) in less than an hour. Fig.9 shows an example of wavelength comparison for sequence's (Fig.12) image-2. Peak wavelengths derived from TS-X image spectra were superimposed on wavelength derived from CWAM model results from peak period and actual local depth. Both, model and TS-X show identical spatial distribution in wavelength domain $60 \mathrm{~m}-100 \mathrm{~m}$ with local differences in order of $10 \mathrm{~m}-20 \mathrm{~m}$.

Another example is one successful acquisition of a storm on 10.12.2014 at 17:19 UTC. A sequence of 6 TS-X StripMap images covers the German Bight completely. The wind increases up to $18 \mathrm{~m} \cdot \mathrm{s}^{-1}$ (XMOD-2) at the position of Lightship "TWEms" provided by BSH (Meteorology: DWD) located at $54.1666 \mathrm{~N}$ and $6.3500 \mathrm{E}$ reported wind around $17.5 \mathrm{~m} \cdot \mathrm{s}^{-1}$. Fig. 13 shows model results of wave model of HZG (COSYNA) at 18:00 UTC and 3-D $H_{S}$ derived from TS-X images using XWAVE_C algorithm. A time delay of 40min does not play a role according to model time series: from 00:00 UTC the wave height increases permanently from $4 \mathrm{~m}$ to about $7 \mathrm{~m}$ until 16:00 and remains constant further on until 11.12.2014. It can be clearly seen that TS-X data reproduce the same spatial patterns including wind dependent growth of sea state (fetch). On the other hand, TS-X has more spatial variability as the smoother model data.
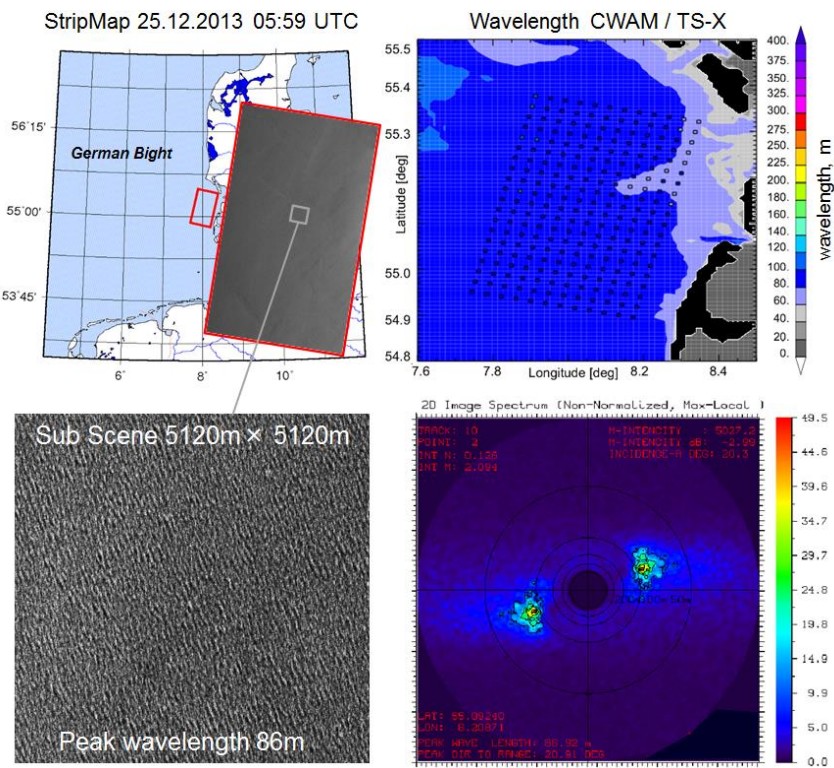

Figure 9. An example of comparison for wavelength derived from TS-X image and from CWAM model results from peak period and actual local depth using dispersion relation. TerraSAR-X wavelength (squares) are superimposed on the model data. 


\section{DISCUSSION}

The collected data show that sea state which propagates in azimuth direction (SAR flight) is minimally disturbed by nonlinear effects and results in spectra with practically no visible cut-off while the sea state moving in range direction (to SAR) is strongly perturbed by non-linear artifacts and defocussing. Often these distortions for near-range moving waves are so strong that the waves itself cannot be seen underneath the streak-structures. Such distorted sea state results in spectra with well-visible "cut-off" with a ratio (noise level inside cut-off)/(noise level outside cut-off) in the order of 3-5. These results show that the strongest impact of nonlinear effects (velocity bunching and defocusing) is due to the radial component of phase velocity of the wave $(=0$ in case of azimuth traveling waves and maximal for range traveling waves $=\cos (\theta) \cdot$ wavelength/period) rather than local orbital wave velocities. Contrary to expectations, Fig.10 demonstrates that the more waves are turned towards the SAR range direction, the more distorted and defocused they are by non-linear effects. Additionally, it can be seen that in coastal areas for short waves with wavelength $<\sim 50 \mathrm{~m}$, a "cut-off" is visible. No azimuth components of traveling waves but only range components can be observed in TS-X SAR images.
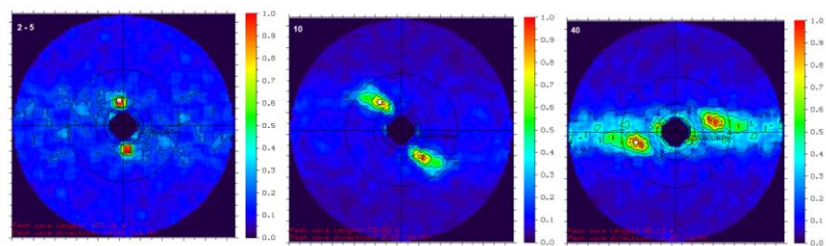

Figure 10. Example for three situations of TerraSAR-X acquisitions for similar sea state traveling in different directions (peak wavelength $\sim 80 \mathrm{~m}-100 \mathrm{~m}$ ): azimuth traveling (left), range traveling (right) and intermediate situation. The azimuth traveling waves are minimally distorted by non-linear effects, the shape of image spectra approaches shape of wave spectra; for $45^{\circ}$ traveling waves the distortions are already visible, and for range traveling waves, the distortions dominate.

\section{SUMMARY AND OUTLOOK}

The algorithms to derive sea state integrated parameters from Xband SAR data were adapted for coastal applications. An NRT version of the Sea State Processor was made operational and processed data were provided for the validation of forecast Wave Model CWAM of German Weather Service in the German Bight in order to improve the predictions in coastal areas and at offshore constructions. It is planned to extend and adapt the algorithms and developed NRT products for Sentinel satellite C-Band SAR data.

\section{ACKNOWLEDGEMENTS}

This study was supported by DLR Space Agency with project DeMARINE-2 SEEGANGSMONITOR and by BMVI (German Federal Ministry of Transport and Digital Infrastructure) with project "Development of Sea-State-Monitor to improve Sea State prediction systems" N 97.0304-2012

The authors are grateful to the team of DLR ground station "Neustrelitz" for active cooperation and organization of NRT service-chain for providing data to users.
The authors gratefully acknowledge COSYNA data portal by HZG, NWS (North West Shelf) Portal by BSH and EMODnet for data providing.

\section{REFERENCES}

Bruck, M. and S. Lehner, 2013. Coastal wave field extraction using TerraSAR-X data, Journal Appl. Remote Sens. 7(1), 073694 .

\section{COSYNA data web portal CODM http://codm.hzg.de/codm/}

Kieser, J., Bruns., T., Behrens, A., Lehner, S. and A. Pleskachevsky, 2013. First Studies with the High-Resolution Coupled Wave Current Model CWAM and other Aspects of the Project Sea State Monitor, Proc. 13th international workshop on wave hindcasting and 4th coastal hazard symposium" (WAVE WORKSHOP), 27 October - 1 November 2013, Banff, Canada.

Lehner, S., Pleskachevsky, A. and M. Bruck, 2012. High resolution satellite measurements of coastal wind field and sea state. International Journal of Remote Sensing, Special Issue: Pan Ocean Remote Sensing: Connecting Regional Impacts to Global Environmental Change, Volume 33, Issue 23, 2012.

Lehner, S., Pleskachevsky, A., Velotto, D., and S. Jacobsen (2013): Meteo-Marine Parameters and Their Variability Observed by High Resolution Satellite Radar Images, Journal of Oceanography 26(2):80-91, http://dx.doi.org/10.5670/ oceanog.2013.36.

Li, X.-M. and S. Lehner, 2013. Algorithm for sea surface wind retrieval from TerraSAR-X and TanDEM-X data, In: Transactions on Geoscience and Remote Sensing.

Pleskachevsky, A., Lehner, S., and W. Rosenthal, 2012. Storm Observations by Remote Sensing and Influences of Gustiness on Ocean Waves and on Generation of Rogue Waves. Ocean Dynamics, Sept. 2012, Volume 62, Issue 9, pp. 1335-1351.

Table 1. Colocation CWAM wave model and TS-X/TD-X StripMap acquisitions: 21 overflights with 64 TS-X images

\begin{tabular}{|c|c|c|c|}
\hline $\begin{array}{l}\text { DWD CWAM } \\
\text { hourly output }\end{array}$ & $\begin{array}{c}\text { TS-X/TDX } \\
\text { scene/passage }\end{array}$ & Pol. & $\begin{array}{l}\text { Number } \\
\text { of images }\end{array}$ \\
\hline 2013-01-24 06:00 & 2013-01-24 05:50 & VV & $\begin{array}{ll}4 \\
\end{array}$ \\
\hline $2013-01-28 \quad 17: 00$ & $2013-01-28 \quad 17: 01$ & $\mathrm{VV}$ & 4 \\
\hline 2013-01-29 06:00 & 2013-01-29 05:59 & VV & 4 \\
\hline 2013-02-04 06:00 & 2013-02-04 05:50 & VV & 2 \\
\hline 2013-02-08 17:00 & 2013-02-08 17:01 & VV & 4 \\
\hline 2013-02-09 06:00 & 2013-02-09 $05: 59$ & VV & 4 \\
\hline 2013-02-19 $\quad 17: 00$ & $2013-02-19 \quad 17: 01$ & $\mathrm{HH}$ & 1 \\
\hline $2013-03-02 \quad 17: 00$ & 2013-03-02 17:01 & $\mathrm{VV}$ & 1 \\
\hline 2013-03-07 17:00 & 2013-03-07 17:10 & VV & 1 \\
\hline 2013-03-09 06:00 & 2013-03-09 05:50 & $\mathrm{HH}$ & 1 \\
\hline 2013-03-18 17:00 & $2013-03-18 \quad 17: 10$ & $\mathrm{HH}$ & 1 \\
\hline 2013-03-20 06:00 & $2013-03-20 \quad 05: 50$ & $\mathrm{VV}$ & 3 \\
\hline 2013-04-05 06:00 & 2013-04-05 $05: 59$ & $\mathrm{HH}$ & 1 \\
\hline 2013-04-09 $17: 00$ & 2013-04-09 $17: 10$ & $\mathrm{HH}$ & 1 \\
\hline 2013-04-11 06:00 & 2013-04-11 05:50 & VV & 1 \\
\hline $2013-11-21 \quad 17: 00$ & 2013-11-21 17:01 & $\mathrm{HH}$ & 4 \\
\hline 2013-12-03 06:00 & $2013-12-03 \quad 05: 59$ & VV & 4 \\
\hline 2013-12-20 $06: 00$ & $2013-12-20 \quad 05: 50$ & $\mathrm{VV}$ & 4 \\
\hline $2013-12-24 \quad 17: 00$ & $2013-12-24 \quad 17: 01$ & $\mathrm{VV}$ & 5 \\
\hline 2013-12-25 $06: 00$ & 2013-12-25 05:59 & $\mathrm{VV}$ & 5 \\
\hline 2013-12-31 06:00 & $2013-12-3105: 50$ & $\mathrm{VV}$ & 4 \\
\hline 2014-01-04 17:00 & 2014-01-04 17:01 & VV & 5 \\
\hline
\end{tabular}




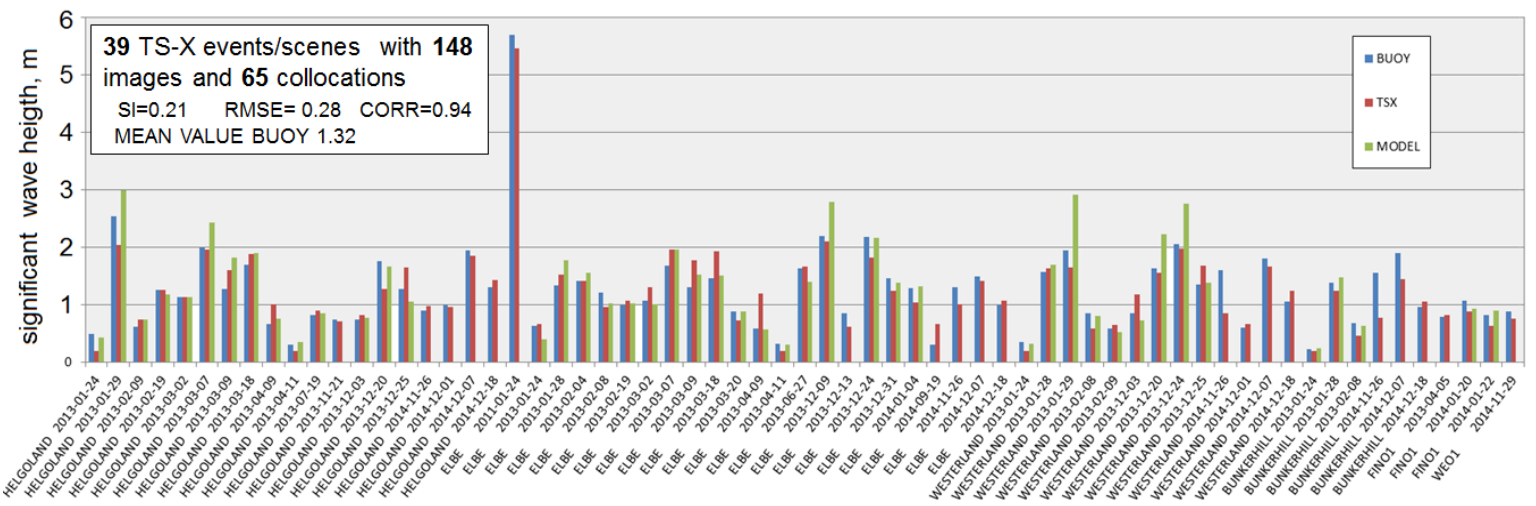

Figure 11. Comparison of total significant wave height $H_{S}$ derived using TerraSAR-X /XWAVE_C algorithm, buoy measurements and collocated CWAM model results for all acquired and collocated TS-X images in German Bight 2013-2014 (65 collocations) including a storm on 09.12.2011. The data are systematized according to measurement station and acquisition time.

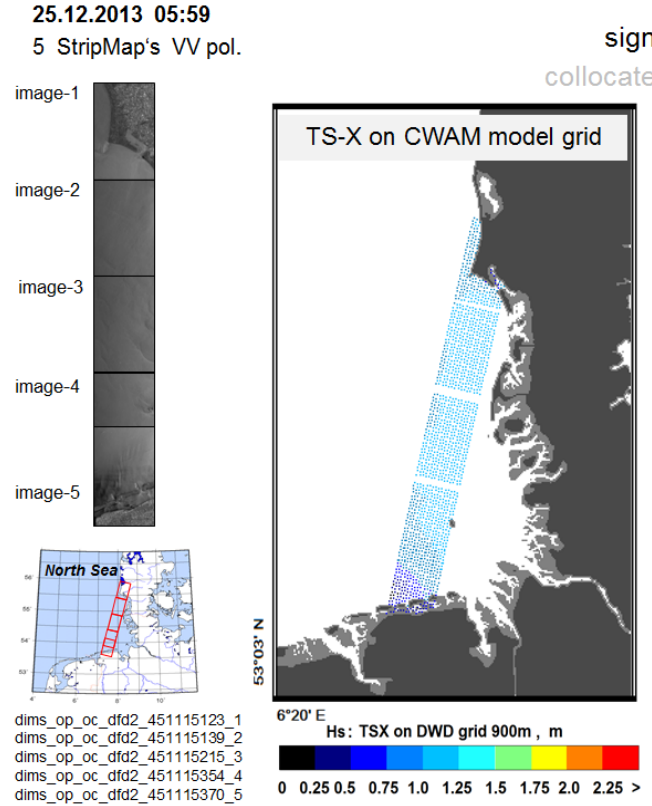

significant wave height Hs
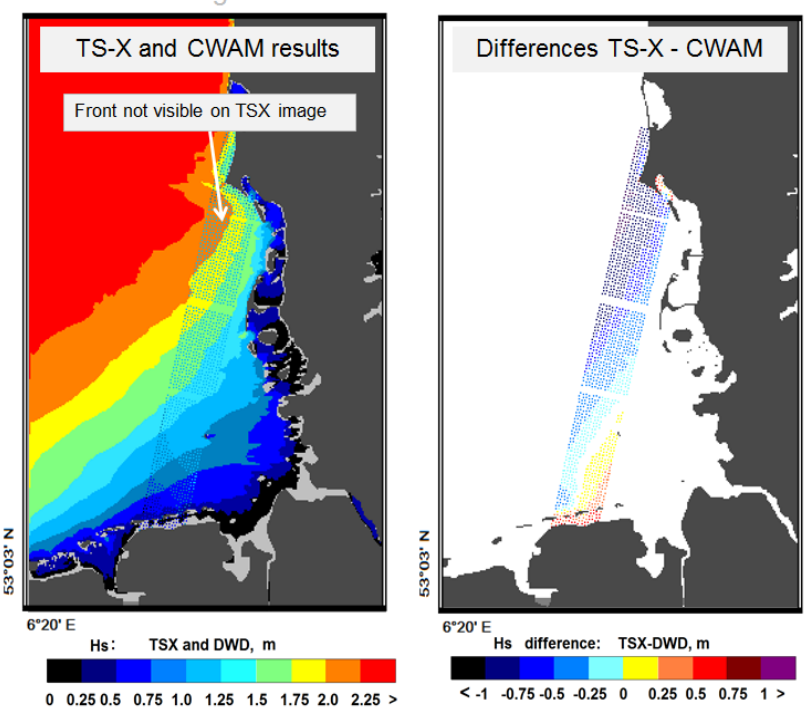

Figure 12. Example of a spatial comparison of sea state derived using TerraSAR-X /XWAVE_C with the CWAM model results. The coastal model shows a front with $H s>2.25 \mathrm{~m}$, which is not visible in TS-X data. The comparison of local wind shows also differences: XMOD-2 local wind from TS-X image is $\sim 8 \mathrm{~m} \cdot \mathrm{s}^{-1}$ and model wind is $12 \mathrm{~m} \cdot \mathrm{s}^{-1}$ in the area of the front.
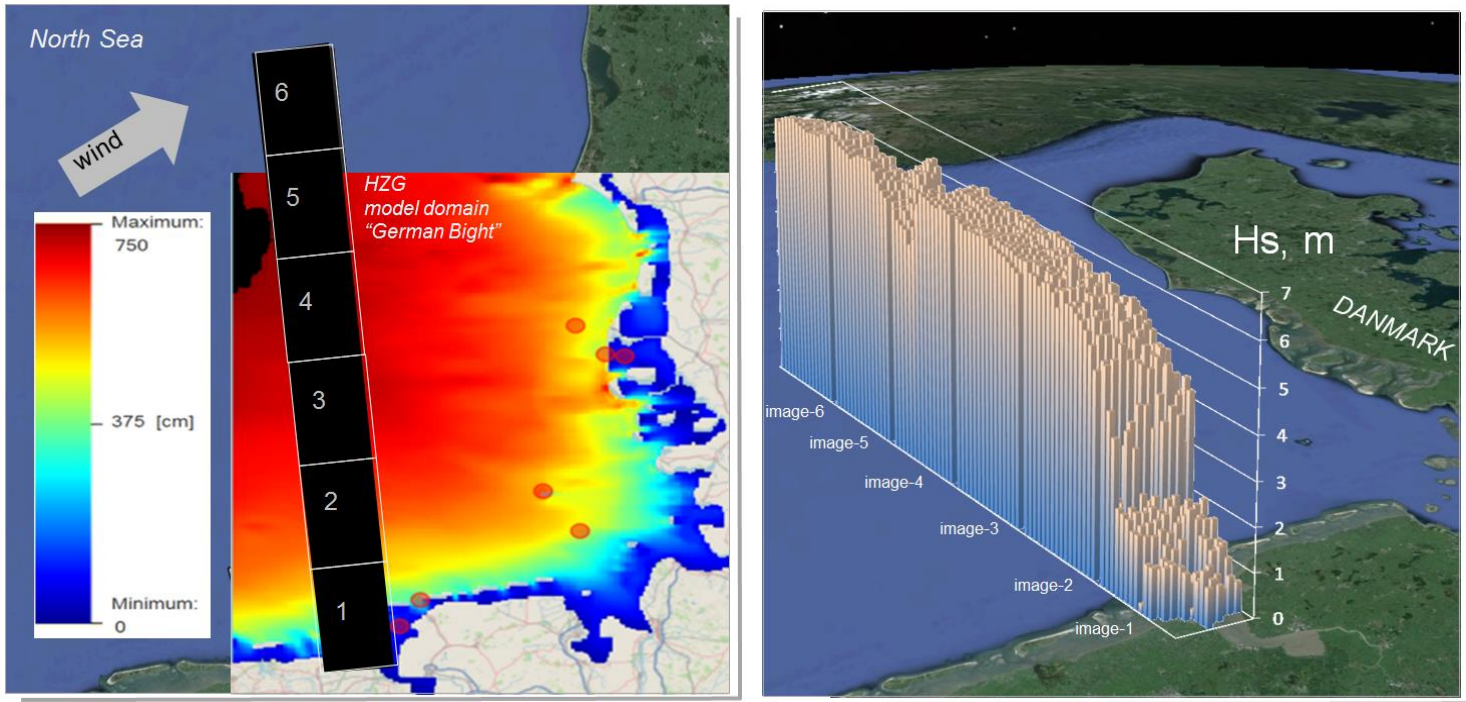

Figure 13. An example of an acquisition of a storm on 10.12.2014 at 17:19 UTC. Sequences of 6 TerraSAR-X StripMap images across North Sea with results of HZG wave model "German Bight" as background (source: COSYNA) (left) and 3-D significant wave height on (C) Google Earth extracted from TS-X images using XWAVE algorithm (right). 